\title{
Ankle Foot Orthosis (AFO) stiffness design for mitigation of ankle inversion injury
}

\author{
D. Teixeira, J. Milho, M. S. Carvalho, M.A.R. Loja
}

\begin{abstract}
Modelling and simulation of human movement has the potential to improve the design of medical devices and rehabilitation process by enabling the identification of causeeffect relationships in individuals suffering from neurological and musculoskeletal issues. The main goal of this work was to provide a simulation-based stiffness design for an Ankle Foot Orthosis (AFO) that can help to mitigate the risk of a sprain by ankle inversion during the landing in freefall which is known to occur for subtalar angles higher than 25 degrees. Computational simulations were performed using human movement models with and without a passive AFO, to access the AFO sensitivity for the translational stiffness that prevents the cuff from translating with respect to the footplate. The Design of Experiments (DoE) methodology was used to access sensitivities between the three principal directions of the AFO stiffness.

Results revealed that the ankle inversion angle was less than 25 degrees when increasingly larger values of translational stiffness were used, although a nonlinear behaviour was observed between the three principal directions of the AFO stiffness, for which injury safe design configurations were obtained.
\end{abstract}

\section{INTRODUCTION}

Modelling and simulation of human movement has the potential to improve the design of medical devices and rehabilitation process by enabling the identification of causeeffect relationships in individuals suffering from neurological and musculoskeletal issues [1]. Dynamic musculoskeletal simulations are becoming an increasingly viable approach to determine how the components of the musculoskeletal system interact in the production of movement. For this purpose, three-dimensional (3D) simulations are required, activated by muscles that accurately reproduce the various steps of gait movement and other movement dynamics of singular individuals. These simulations allow to identify which elements are causing movement alteration, for example, bone deformities, abnormal muscle excitations or muscular weakness. Neuro-musculoskeletal system models

D. Teixeira is with ISEL, Instituto Superior de Engenharia de Lisboa, Instituto Politécnico de Lisboa, Av. Conselheiro Emídio Navarro 1, 1959007 Lisboa, Portugal. (e-mail: daniela.filipa.silva.teixeira@gmail.com).

J. Milho is with GIMOSM - Grupo de Investigação em Modelação e Optimização de Sistemas Multifuncionais, ISEL - Instituto Superior de Engenharia de Lisboa, Instituto Politécnico de Lisboa, Av. Conselheiro Emídio Navarro 1, 1959-007 Lisboa, Portugal, and with LAETA, IDMEC, Instituto Superior Técnico, Universidade de Lisboa, Av. Rovisco Pais, 1049-001 Lisboa, Portugal (e-mail: joao.milho@dem.isel.ipl.pt)

M. S. Carvalho is with UNIDEMI, Faculdade de Ciências e Tecnologia, Universidade NOVA de Lisboa, Portugal (email: marta.verdete@fct.unl.pt).

M.A.R. Loja is with GIMOSM - Grupo de Investigação em Modelação e Optimização de Sistemas Multifuncionais, ISEL / IPL - Instituto Politécnico de Lisboa, and with IDMEC/IST - Instituto de Engenharia Mecânica, Av. Rovisco Pais, 1049-001 Lisboa, Portugal (email: amelialoja@tecnico.ulisboa.pt). provide information on internal muscle forces and joint loads for a wide variety of scenarios, such as walking, running, and jumping. Understanding how muscular forces coordinate movement is very important for the application of assistive devices, planning rehabilitation treatments and understanding the fundamental principles of human locomotion. The use of simulations allows, therefore, the realization of studies to test hypotheses, predict functional outcomes and identify emerging behaviours $[2,3]$.

Orthoses and prostheses are auxiliary medical devices to help people with disabilities. Orthoses are biomechanical devices often prescribed to support, realign or redistribute pressures and modify structural and functional characteristics of human neuromuscular and musculoskeletal systems. These devices apply forces on the body, whose amount, place of application and control contribute to their effectiveness $[4,5]$. The production of this type of orthopedic devices is based, even today, on craft skills, in which the processes involved are time consuming and require experienced professionals who generally take their decisions based on experience and trial-error, rather than resort to principles based on scientific and engineering evidence. In addition to this aspect, the mechanical properties of the traditionally produced apparatus are only roughly estimated, which may result in poor rehabilitation designs and processes for the patient in question [5]. Passive AFO is a biomechanical device (articulated or not) that do not have any type of electronic controller but may contain mechanical elements such as springs or shock absorbers to control the movement of the joint of the ankle during gait. This is the type of AFO often prescribed to treat issues related to injuries in the ankle complex.

In clinical practice it is inconvenient to find the ideal AFO for a given patient testing several orthoses with different characteristics, namely stiffness and flexibility. Thus, currently, simulation models are already used to explore the influence of this parameter without requiring the constant presence of the individual [6]. It is important to note that the flexibility of an ankle and foot orthosis depends on the characteristics of its design, such as the thickness of the wall of the AFO, the components around the ankle, the stiffness, its contours, etc. Thus, to improve the performance of the gait movement these incorporate a wide range of mechanical characteristics that depend both on the selection of materials and their geometry [7, 8]. Mechanical properties can be altered during the process of manufacturing and adaptation of the orthosis, for example, there may be changes in the cutting lines or in the definition of the "neutral" position of the same, but always to meet the individual needs [8]. 
The traumatic injury of the lateral ligaments of the ankle, mainly of the anterior peronium-astragalian ligament and the fibula-calcaneal ligament, is one of the most common lesions occurring in the lower limb, about $77 \%$ of the cases, and is caused by excessive inversion or supination. These injuries occur more frequently during sporting activities, particularly in those involving running or walking on irregular terrain. Given the high number of participants in this type of activities, there is a growing need to develop improved diagnostic and treatment strategies for such lesions [9, 10]. The subtalar joint is formed by the upper lip of the astragalus and by the calcaneus, creating two separate articular cavities. This articulation allows the inversion and eversion movements, or supination and pronation. Based on the articular amplitude corresponding to the inversion movement, it can be affirmed that inversion angles greater than 25 degrees may cause injury by inversion of the ankle, this value being the one that will be used as a basis for conducting this investigation $[10,11]$.

In the present work, to mitigate the risk of sprain when ankle inversion angles are higher than 25 degrees, a simulation-based methodology was implemented for the stiffness design of an Ankle Foot Orthosis.

\section{MATERIALS AND METHODS}

The modelling and simulation of the human movement performed in this work was based on the models available in the software OpenSim [12]. This free access software was created for modelling, simulation and analysis of the neuromusculoskeletal system, and includes low-level computational tools that are invoked by an application, also possessing a graphical user interface (GUI). This software has been developed on core computational components that allows to derive equations of motion in dynamic systems, perform numerical integration and solve restricted problems of nonlinear optimization, also enabling to generate dynamic movement simulations from experimentally collected data. A model in OpenSim represents the dynamics of a system of rigid bodies and joints that are triggered by forces to produce a movement. The model consists of parts of the musculoskeletal system: body segments, joints, forces, markers, constraints and restrictions, contact geometry and controllers. The skeletal part is represented by rigid bodies connected by joints that define how a segment moves in relation to the segment that articulates to this proximally. Constraints can be applied to limit the movement of bodies. The muscles are modelled as specialized strength elements that act on muscular points (insertion and origin points) and are connected to rigid bodies. The models may also possess other types of forces that are applied externally, as is the case of ground reaction forces, passive structures (ligaments) and linear and torsion actuators. The muscles provide information to calculate muscle-to-actuator lengths and velocities, and typically include the dynamics of activation and muscle contraction.

The biomechanical model used in this work corresponds to a 3D model with 23 degrees of freedom available online from the OpenSim libraries, with a mass of $75.2646 \mathrm{~kg}$ or $78.0146 \mathrm{~kg}$ when considering the model without or with the AFO, respectively. The biomechanical model without the
AFO is composed of the body segments of the trunk, pelvic girdle and two lower limbs according to figure 1, which also represents the initial position configuration for the simulations. This model presents a total of 12 rigid bodies, 70 muscle-tendon units (muscles) and 12 intersegmental joints.

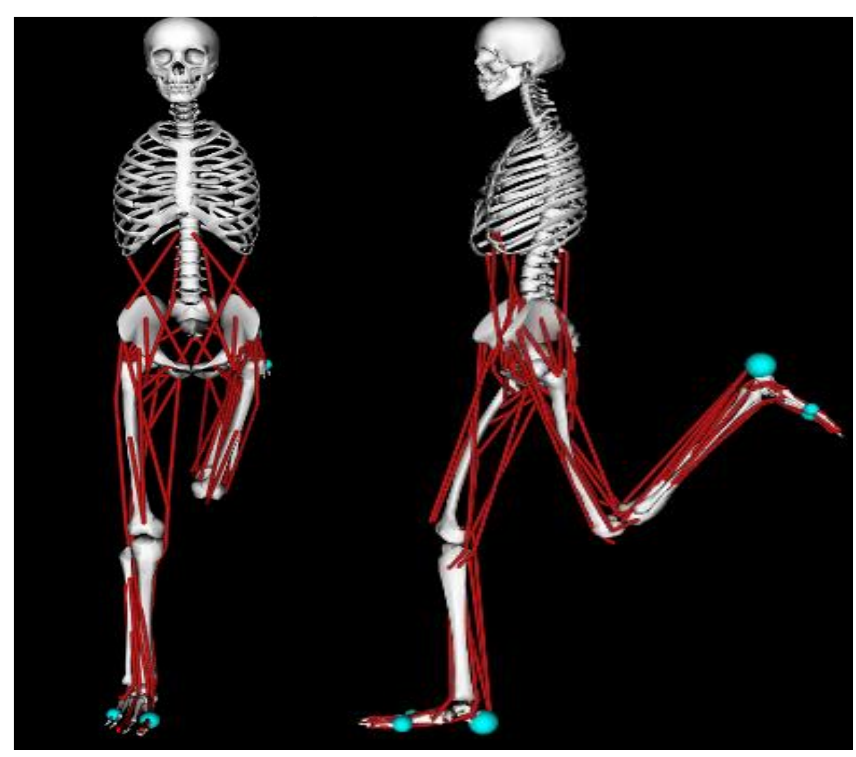

Figure 1. Biomechanical model at initial position configuration.

The simulation scenario is established considering a landing in free fall of the model on a 20 degrees inclined plane, in which it will be possible to evaluate the risk of a lesion by inversion of the ankle, based on the estimation of the subtalar angle. To represent the landing surface, the simulation includes a fixed contact plane located below the musculoskeletal model. This contact platform interacts with the feet of the model that has contact spheres with the objective of producing contact forces between the foot and the platform.

For biomechanical models, in general, that involve contact between the components of the model, these contacts, in many cases are idealized by joints or restrictions. However, the real contact forces arise from the local deformation in the interface between the materials in contact that compose the biological systems. In the case of this model, the approach to the question of contact forces is carried out considering normal and tangential forces between rigid bodies during contact, applying a model that treats deformation/impact as a continuous event. It is important to consider that the impact velocity should be low, and the material of the biological structures has viscoelastic behaviour, so the deformation is high and the coefficients of restitution (dissipated kinetic energy) are expected to be low (lower than 0.5). It is also important to ensure that contact forces, in the absence of deformation, are zero [13]. Regarding the present model, figure 2 presents an example of two time frames during the free fall simulation, corresponding to pre and post contact of the foot with the inclined landing platform. 


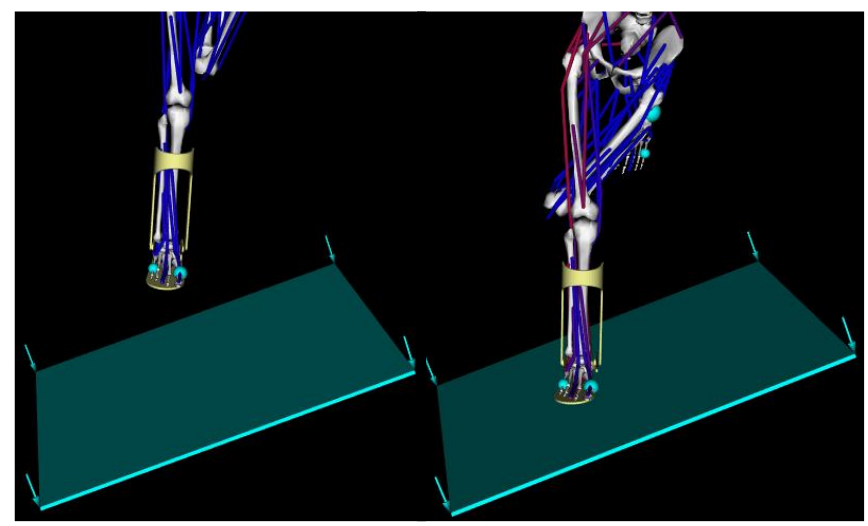

Figure 2. Pre and post contact of foot with inclined plane (example).

Evaluation of the risk of a lesion by inversion of the ankle during the free fall is based on the analysis of the simulation results for the biomechanical model subtalar angle. An example of the subtalar angles during a time interval of 0,4 seconds of simulation is presented in figure 3. The risk of injury is defined above the subtalar angle of 25 degrees, which in the example presented in figure 3 occurs after 0,34 seconds. Conversely, subtalar angles below 25 degrees are considered injury safe.

Within the optimization theory, a simulation is a series of tests in which the input variables are changed according to a given rule to identify the reasons for the changes in the output response [14]. The method Design of Experiments (DoE) consists of a strategy designed to minimize the number of analyses needed to identify the multidimensional space, and its objective is to produce n-dimensional surfaces with soft characteristics for better understanding the variability of the function with the project variables. This methodology has been applied to access biomechanical injury indices in vehicle collision scenarios, based on the Central Composite Design (CCD) [15].

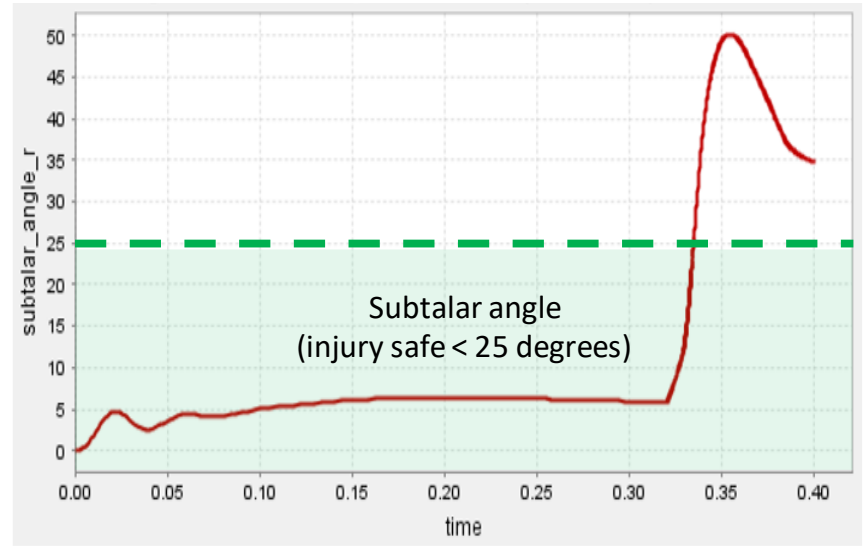

Figure 3. Time evolution for subtalar angle (example) and injury safe zone below 25 degrees.

The complete process requires a number of points defined as a CCD cell to be identified; that the quadratic response surface coefficients are calculated to identify the superficial response function; and the optimization problem is solved by considering the function of surface response representative of the detailed response of the model. The CCD defines the coordinates of the $\mathrm{k}$-encoded variables required to perform $\mathrm{n}$ numerical simulations that allow the identification of the quadratic response surface. In the case of present work, $\mathrm{k}=3$ corresponds to the 3 project variables considered, one for each stiffness direction $x, y$ and $z$, representing the translational stiffness that prevents the AFO cuff from translating with respect to the footplate, as presented in figure 4. In these conditions, DoE establishes $n=15$ simulations required to be performed to identify the quadratic response surface. The coordinates of the coded variables to be used in each simulation are defined based on the values of $n$ and $k$. Additionally, considering the values for the reference design variables and their range of variation, it is possible to calculate the project variables to be used in each numerical simulation.

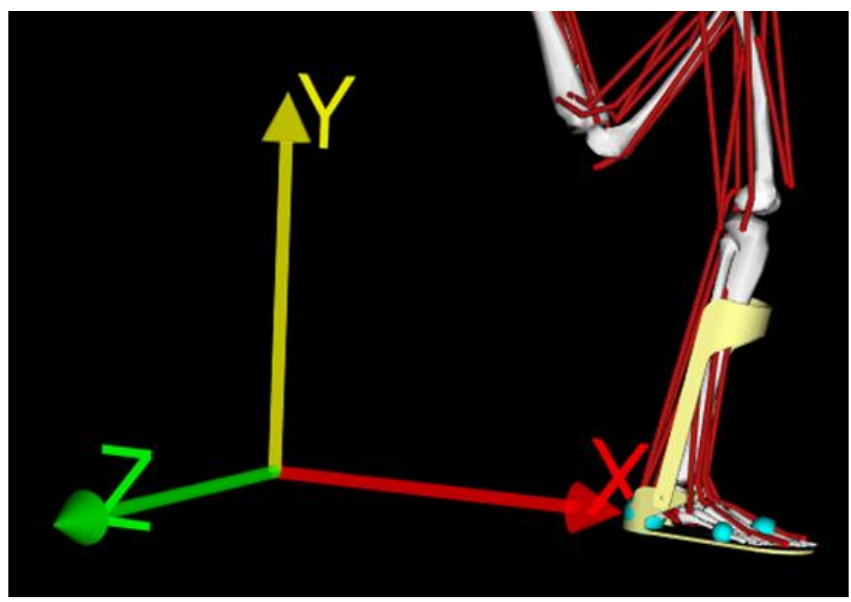

Figure 4. Translational stiffness directions $\mathrm{x}, \mathrm{y}$ and $\mathrm{z}$.

\section{RESUlTS AND Discussion}

To understand the weight of the AFO translational stiffness along $\mathrm{x}, \mathrm{y}$ and $\mathrm{z}$ directions in the variation of the value of the inversion angle corresponding to the subtalar angle, the 15 simulations required by the DoE methodology were performed.

The analysis of these simulations allowed identifying feasible values for the AFO stiffness parameters that maintain subtalar angles values below 25 degrees, which corresponds to the injury risk free range.

The optimization study was performed considering that the three project variables correspond to $\mathrm{x}, \mathrm{y}$, and $\mathrm{z}$ values of translational stiffness. The corresponding results for the 15 simulations of the DoE regarding the three translational stiffness variables and corresponding subtalar maximum angle are presented in table I. The simulations corresponding to injury risk safe configurations with maximum subtalar angle below 25 degrees, are marked with an asterisk (*) in table I.

The results of this DoE show that the higher the value of translational stiffness, the lower the inversion angle. Data in table I reveal that multiple sets of values for each translational stiffness in directions $\mathrm{x}, \mathrm{y}$, and $\mathrm{z}$, can achieve 
subtalar angles less than 25 degrees and which combinations allow achieving this goal. Based on this information, insight can be provided for the design of personalized AFO orthosis, based on the obtained directional stiffness requirements and corresponding movement constraints.

The methodology presented in this work can be further explored to include additional design variables that may include among others, the AFO mass, the muscle activation parameters, or the motor torque for active AFO devices.

TABLE I. DOE SIMULATION RESULTS

\begin{tabular}{|c|c|c|c|c|}
\hline $\begin{array}{c}\text { Simulation } \\
\text { number }\end{array}$ & $\begin{array}{c}X \\
\text { translational } \\
\text { stiffness } \\
(\mathbf{N} / \mathbf{m})\end{array}$ & $\begin{array}{c}\mathbf{Y} \\
\text { translational } \\
\text { stiffness } \\
(\mathbf{N} / \mathbf{m}) \\
\end{array}$ & $\begin{array}{c}Z \\
\text { translational } \\
\text { stiffness } \\
(\mathbf{N} / \mathbf{m}) \\
\end{array}$ & $\begin{array}{c}\text { Subtalar } \\
\text { angle } \\
\text { (degrees) }\end{array}$ \\
\hline 1 & 34391.2 & 34391.2 & 34391.2 & 34.1 \\
\hline 2 & 120608.8 & 34391.2 & 34391.2 & 26.2 \\
\hline 3 & 34391.2 & 120608.8 & 34391.2 & $23.0^{*}$ \\
\hline 4 & 120608.8 & 120608.8 & 34391.2 & $19.3^{*}$ \\
\hline 5 & 34391.2 & 34391.2 & 120608.8 & 32.8 \\
\hline 6 & 120608.8 & 34391.2 & 120608.8 & 25.7 \\
\hline 7 & 34391.2 & 120608.8 & 120608.8 & $22.8^{*}$ \\
\hline 8 & 120608.8 & 120608.8 & 120608.8 & $19.2^{*}$ \\
\hline 9 & 5000.0 & 77500.0 & 77500.0 & 29.6 \\
\hline 10 & 150000.0 & 77500.0 & 77500.0 & $20.8^{*}$ \\
\hline 11 & 77500.0 & 5000.0 & 77500.0 & 33.7 \\
\hline 12 & 77500.0 & 150000.0 & 77500.0 & $19.1^{*}$ \\
\hline 13 & 77500.0 & 77500.0 & 5000.0 & $24.6^{*}$ \\
\hline 14 & 77500.0 & 77500.0 & 150000.0 & $24.1^{*}$ \\
\hline
\end{tabular}

* injury risk safe configurations.

\section{ACKOWLEDGMENTS}

For second and fourth authors, this work was supported by Foundation for Science and Technology (FCT), through IDMEC, under LAETA [project number UID/EMS/50022/2019]. For the third author, this work was supported by FCT under the project UNIDEMI [project number UID/EMS/00667/2019].

\section{REFERENCES}

[1] Seth, A., Sherman, M., Reinbolt, J. A., \& Delp, S. L., OpenSim: A musculoskeletal modeling and simulation framework for in silico investigations and exchange. Procedia IUTAM, 2, 212-232, 2011.
[2] Hicks, J. L., Uchida, T. K., Seth, A., Rajagopal, A., \& Delp, S. L., Is My Model Good Enough? Best Practices for Verification and Validation of Musculoskeletal Models and Simulations of Movement. Journal of Biomechanical Engineering, 137(2), 20905-1-20905-24, 2015.

[3] Reinbolt, J. A., Seth, A., \& Delp, S. L., Simulation of human movement: Applications using OpenSim. Procedia IUTAM 2, 2, 186198, 2011.

[4] Jin, Y. A., Plott, J., Chen, R., Wensman, J., \& Shih, A., Additive manufacturing of custom orthoses and prostheses - A review. Procedia CIRP, 36, 199-204, 2015.

[5] Pallari, J. H. P., Dalgarno, K. W., Munguia, J., Muraru, L., Peeraer, L., Telfer, S., \& Woodburn, J., Design and additive fabrication of foot and ankle-foot orthoses. In 21 st Annual International Solid Freeform Fabrication Symposium - An Additive Manufacturing Conference, SFF 2010 (pp. 834-845), 2010.

[6] Bregman, D. J. J., van der Krogt, M. M., de Groot, V., Harlaar, J., Wisse, M., \& Collins, S. H., The effect of ankle foot orthosis stiffness on the energy cost of walking: A simulation study. Clinical Biomechanics, 26(9), 955-961, 2011.

[7] Harper, N. G., Esposito, E. R., Wilken, J. M., \& Neptune, R. R., The influence of ankle-foot orthosis stiffness on walking performance in individuals with lower-limb impairments. Clinical Biomechanics, 29(8), 877-884, 2014.

[8] Hegarty, A. K., Petrella, A. J., Kurz, M. J., \& Silverman, A. K., Evaluating the Effects of Ankle-Foot Orthosis Mechanical Property Assumptions on Gait Simulation Muscle Force Results. Journal of Biomechanical Engineering, 139(3), 31009-1-8, 2017.

[9] Fong, D. T.-P., Chan, Y.-Y., Mok, K.-M., Yung, P. S., \& Chan, K.M., Understanding acute ankle ligamentous sprain injury in sports. Sports Medicine, Arthroscopy, Rehabilitation, Therapy \& Technology, 1, 14, 2009.

[10] Siegler, S., Chen, J., \& Schneck, C. D., The Effect of Damage to the Lateral Collateral Ligaments on the Mechanical Characteristics of the Ankle Joint-An In-Vitro Study. Journal of Biomechanical Engineering, 112(2), 129-137, 1990.

[11] Lapointe, S. J., Siegler, S., Hillstrom, H., Nobilini, R. R., Mlodzienski, A., \& Techner, L., Changes in the flexibility characteristics of the ankle complex due to damage to the lateral collateral ligaments: An in vitro and in vivo study. Journal of Orthopaedic Research, 15(3), 331341, 1997.

[12] Delp, S. L., Anderson, F. C., Arnold, A. S., Loan, P., Habib, A., John, C. T., Thelen, D. G., OpenSim : Open-Source Software to Create and Analyze Dynamic Simulations of Movement. IEEE Transactions on Biomedical Engineering, 54(11), 1940-1950, 2007.

[13] Lankarani, H. M., \& Nikravesh, P. E., A Contact Force Model With Hysteresis Damping for Impact Analysis of Multibody Systems. Journal of Mechanical Design, 112(3), 369-376, 1990.

[14] Cavazzuti, M., Design of Experiments. Optimization Methods: From Theory to Design Scientific and Technological Aspects in Mechanics. Berlin, Heidelberg: Springer Berlin Heidelberg, 2013.

[15] Carvalho, M., Milho, J., Ambrosio, J., Ramos, N., Railway occupant passive safety improvement by optimal design. International Journal of Crashworthiness, 22(6), 624-634, 2017. 